\title{
New refinement strategies for pseudoatom databank - towards wider range of application
}

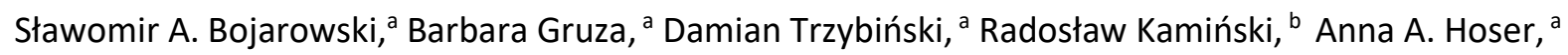 \\ Prashant Kumar, ${ }^{a}$ Krzysztof Woźniak $^{a}$ and Paulina M. Dominiak ${ }^{\text {a }}{ }^{*}$ \\ ${ }^{a}$ Biological and Chemical Research Center, Department of Chemistry, University of Warsaw, ul. Żwirki \\ i Wigury 101, 02-089 Warszawa, Poland. E-mail: pdomin@chem.uw.edu.pl \\ ${ }^{\mathrm{b}}$ Department of Chemistry, University of Warsaw, Żwirki i Wigury 101, 02-089 Warsaw, Poland
}

\section{Abstract}

Pseudoatom databanks, collecting parameters of multipole model of electron densities for various atom types, are used to replace Independent Atom Model by the more accurate Transferable Aspherical Atom Model (TAAM) in crystal structure refinement. They may also be used to reconstruct electron density of a molecule, crystal or biomacromolecular complex in fast yet quite accurate way and compute from it various properties, like energy of electrostatic interactions, for example. Even faster, but similarly accurate in electrostatic energy estimations model exists, the aug-PROmol. Model analogous to aug-PROmol cannot be built from the current pseudoatom databanks, as they perform badly when truncated to the monopole level. Here we searched for new strategies of multipole model refinements, leading to better parametrization already at the monopole level. This would allow to create in a single route of model parametrization a pseudoatom databank, which would be suitable for both crystal structure refinement and rapid electrostatic energy calculations. Such a route does not exist yet, because as we show here, the aug-PROmol model, alternative to the current pseudoatom databanks, is not suitable for crystal X-ray structure refinement.

Here we show that cumulative approach to multipole model refinements, as oppose to simultaneous or iterative refinements of all multipole model parameters $\left(P_{v}, \kappa, P_{l m}, \kappa^{\prime}\right)$ leads to substantially different models of electron density. Cumulative refinement of $P_{l m}$ first and then $\kappa^{\prime}$ parameters is much worse than simultaneous. It results in electron density model giving wrong estimates of electrostatic interaction energies and atomic displacement parameters. Cumulative refinement of two blocks of parameters, $P_{v}$ and $\kappa$ first and then $P_{l m}$ and $\kappa^{\prime}$, on the other hand, leads to the $P_{v} \kappa \mid P_{I m} \kappa^{\prime}$ model having promising properties. It is similarly good as University at Buffalo DataBank (UBDB) of pseudoatoms in X-ray structure TAAM refinement and electrostatic energy estimations, especially for less polar molecules. When truncated to monopole level, the $P_{v} \kappa$ model has a chance to replace the aug-PROmol in fast yet accurate electrostatics energy calculations, although some improvements in $\kappa$ parametrization for polar functional groups are still needed. The $\mathrm{P}_{\mathrm{v}} \kappa \mid \mathrm{P}_{\mathrm{Im}} \kappa^{\prime}$ model is also a source of point charges which behave similarly to the RESP charges in electrostatic interaction energy estimations.

\section{Introduction}

X-ray beams are scattered mostly at electrons. Thus X-ray diffraction experiments provide information on electron densities, $\rho(\mathbf{r})$, in the solid state. Since the physical properties of molecules are functionals of their electron densities, the accurate knowledge of $\rho(\mathbf{r})$ is crucial for a wide variety of chemical, physical, and even biological studies. Parallel to the progress in instrumentations, there 
have been a great development in theoretical approaches to enhance the quality and the type of information to be extracted from $\mathrm{X}$-ray diffraction data. As a result, over past decades, more and more complex models of electron densities were established.

One of the very first and the most robust model is Independent Atom Model (IAM). In IAM the electron density of the molecule is approximated in terms of a promolecule. It means, the modelled total electron density is represented by superposition of spherical densities of isolated atoms $\rho_{\text {atom }}^{0}\left(\boldsymbol{r}-\boldsymbol{R}_{\text {atom }}\right)$ centered at $\boldsymbol{R}_{\text {atom }}$ :

$$
\rho_{\text {IAM }}(\boldsymbol{r})=\sum_{\text {atom }} \rho_{\text {atom }}^{0}\left(\boldsymbol{r}-\boldsymbol{R}_{\text {atom }}\right)
$$

The model is following the crucial concept in chemistry: partitioning the electron density into its atomic contributions. The IAM was very successful in the last century, especially in structural crystallography.

Models more advanced than IAM are also in use (De Bruyne \& Gillet, 2020; Hansen \& Coppens, 1978; Jayatilaka \& Dittrich, 2008; Jayatilaka \& Grimwood, 2001; Lübben et al., 2019; Malaspina et al., 2019; Stewart, 1976; Tanaka, 2018). Among them is the Hansen - Coppens multipole model (HCMM) (Hansen \& Coppens, 1978) which is no longer spherical and allows to model charge transfer between atoms in molecules and crystals. The electron density of an atom (called pseudoatom) is defined in the HCMM as follows

$$
\rho_{\text {atom }}(\boldsymbol{r})=P_{c} \rho_{\text {core }}(r)+P_{v} \kappa^{3} \rho_{\text {valence }}(\kappa r)+\sum_{l=0}^{4} \sum_{m=-l}^{l} \kappa_{l}^{\prime 3} R_{l}\left(\kappa_{l}^{\prime} r\right) P_{l m} d_{l m}(\theta, \varphi)
$$

where the $\rho_{\text {core }}(\mathbf{r})$ and $\rho_{\text {valence }}(\mathbf{r})$ are electron densities of core and valence electrons, respectively, precomputed for isolated atoms and scaled to one electron. The third part of the model, built from Slater radial functions $R_{l}(r)$ and spherical harmonics $d_{l m}(\theta, \varphi)$ are designed to describe deformations of valence electron densities. $P_{c}, P_{v}$ and $P_{l m}$ are electron population parameters and $\kappa$ and $\kappa^{\prime}$ are expansion-contraction parameters. In standard applications of HCMM $P_{v}, \kappa, P_{l m}$ and $\kappa^{\prime}$ parameters are refined against diffraction data, $P_{c}$ is kept frozen at formal values characteristic for particular chemical elements, monopole term $(l=0)$ in the deformation part is not used thus $P_{v}$ and $\kappa$ parameters are the only responsible for modelling monopoles, and $\kappa^{\prime}$ parameters for particular levels of multipolar expansion (from $l=1$ to $l=4$ ) are kept constrained to each other thus effectively there is only one $\kappa^{\prime}$ parameter per pseudoatom. The diffraction data must be of high quality and of subatomic high resolution to allow for stable refinement, proper deconvolution of electron density from atomic motions, and to maintain statistically valid ratio of number of reflections per refined parameter. Only a small part of the experimental diffraction data fulfil such criteria.

The parameters of HCMM have specific values for atoms with a similar chemical environment (Brock et al., 1991). This observation made it possible to build pseudoatom databanks (Zarychta et al., 2007; Domagała et al., 2012; Nassour et al., 2017; Dittrich et al., 2004, 2005, 2006, 2013; Volkov et al., 2007; Dominiak et al., 2007; Jarzembska \& Dominiak, 2012; Kumar et al., 2019). The databanks collect HCMM parameters derived from the multipole model refinements based on experimental diffraction data (Zarychta et al., 2007; Domagała et al., 2012; Nassour et al., 2017) or theoretical simulations (Dittrich et al., 2004, 2005, 2006, 2013; Volkov et al., 2007; Dominiak et al., 2007; Jarzembska \& Dominiak, 2012; Kumar et al., 2019). Theoretical simulations allowed the parametrization of HCMM for a much wider range of different molecules, overcoming the limited access to high-quality experimental data with subatomic resolution.

Pseudoatom databanks have two major areas of application. They are used to replace IAM by more accurate Transferable Aspherical Atom Model (TAAM) in crystal structure refinement (Jha et al., 2020; Gruza et al., 2020; Dittrich et al., 2013; Dadda et al., 2012; Bąk et al., 2011). TAAM parametrized 
with values transferred from a pseudoatom databank allows to refine crystal structure with the same number of refined parameters as IAM, yet achieving better fit of the model to the data and more accurate structure. The databanks may also be used to reconstruct electron density of a molecule, crystal or biomacromolecular complex in fast yet quite accurate way and compute from it various properties, like electrostatic potential, electric field gradient, topological bonding descriptors, or energy of electrostatic interactions (Holstein et al., 2012; Malińska et al., 2014; Mandal et al., 2020; Zarychta et al., 2015).

University at Buffalo DataBank (UBDB) of pseudoatoms (Volkov et al., 2007; Dominiak et al., 2007; Jarzembska \& Dominiak, 2012; Kumar et al., 2019), recently remodeled into the databank of Multipolar Atom Types from Theory and Statistical clustering (MATTS) (Jha et al., 2020), was extensively tested for its applications in crystal structure refining (Jha et al., 2020; Gruza et al., 2020) and electrostatic energy estimating (Kumar et al., 2014; Bojarowski et al., 2017). Detailed analyses based on the S66 and S66x8 benchmark datasets (Řezáč et al., 2011a; b) revealed that combination of UBDB with the Exact Potential / Multipole Moments (EPMM) method of computing electrostatic energies (Volkov, et al, 2004) leads to energies of chemical accuracy at wide range of intermolecular distances and type of interactions. The strength of UBDB+EPMM approach lies mostly not in taking into account higher moments of atomic charge distributions, but in taking into account charge penetration contribution to the energy (Bojarowski et al., 2017).

Question raised if calculation of electrostatic energies might be even faster, even for a cost of slight lost in accuracy. One way to go is to lower the level of multipole expansion, preferable up to monopole term only (Nassour et al., 2017), but still keeping continuous representation of charge density for proper estimation of penetration contributions. It appeared that truncation of multipole expansion of UBDB model to monopole increased the level of errors in the electrostatic energy estimations to not acceptable values (Bojarowski et al., 2017). New model of electron density, however, was introduced, aug-PROmol (Bojarowski et al., 2016). The aug-PROmol model is a hybrid model build upon the HCMM, where the third part is omitted, $\kappa$ for non-hydrogen atoms are kept at 1.0 value and for hydrogen at 1.16 , and $P_{v}$ is computed from the RESP point charges (RESP PC). The RESP point charges are derived externally, on demand for a molecule in question, following procedures widely used in the molecular mechanics field. Alternatively, RESP charges can be taken from the Invariom Point Charges database (Bojarowski et al., 2018). The aug-PROmol model is spherical by design what allows to speed up energy calculation by omitting time consuming integration. The accuracy of electrostatic energy estimation by aug-PROmol+EPMM is similar to the UBDB+EPMM approach. The lack of higher electron density moments might be, however, limitation of the augPROmol model in crystal structure refinement if used instead of IAM. Verification of this hypothesis is a part of investigations presented in this work.

Ideally one would like to have a model: (a) parametrized in one single way, (b) accurate enough for both approaches, TAAM refinement and intermolecular electrostatic interaction energy calculations, and (c) which could be truncated at any level of multipole expansion to speed up energy calculations but with only slight gradual loss in accuracy. One way to go is the complete redesign of HCMM, by adding additional radial functions (Koritsanszky et al., 2011), for example, which would allow the model to describe electron densities in more details and may also impact convergence of the model. Nonetheless, with the current HCMM there is still a room for improvements in the way how it is refined.

Here we designed and tested new strategies for multipole model refinement used while the UBDB databank is created. We focused the most on refinement of monopole functions, to be more specific, on refinement of spherical valence density term. We aimed to enhance the role of spherical 
(monopole) term in electron density modelling. In the standard UBDB/MATTS approach, spherical valence densities were refined simultaneously with angular deformation functions. Here we decided to apply cumulative approach and refine multipole model parameters step by step. We firstly refined only spherical valence densities, allowing them to model atomic electron densities as much as possible. In the next stage(s) of the refinement, we kept spherical valence densities frozen and refined only angular deformation functions. Second crucial decision was related to $\kappa$ and $\kappa^{\prime}$ parameters which model expansion-contraction of electron density functions. These parameters are the most difficult parameters to be refined within the Hansen-Coppens multipole model, should stay close to starting 1.0 values, and influence the values of penetration contributions to electrostatic interaction energies computed from the model. In new refinement strategies we refined $\kappa$ and $\kappa^{\prime}$ parameters either simultaneously with population parameters or independently from them.

The models resulting from new strategies were then firstly validated towards their performance in electrostatic interaction energy estimation, secondly as a source of atomic X-ray scattering factors in crystal structure refinements on experimental data. As in our previous publications (Bojarowski et al., 2016; 2017; 2018; Kumar et al., 2014), the S66 and S66×8 datasets were chosen as a benchmark for the electrostatic energy estimations. In addition to electrostatic energies obtained from the DFT-SAPT approach, the electrostatic energies estimated from the UBDB and augPROmol models constituted the reference points in our validation procedure. To test models in TAAM refinements we collected sub-atomic high-resolution X-ray diffraction data for two molecular crystals: triptycene and cyanuric acid dihydrate. In addition we collected neutron diffraction data for triptycene. Full multipole model refinement on X-ray data, and structural model from neutron diffraction data served as us a reference points for testing new models.

\section{Methods}

\subsection{Electrostatic interaction energy validation on the $\mathrm{S} 66$ and $S 66 \times 8$ datasets}

\subsubsection{Parametrization of electron density models}

New electron density models were obtained for 14 different molecules for geometries extracted from the $S 66$ and $\$ 66 \times 8$ datasets (Řezáč et al., 2011a; b).The parametrization followed the same procedure as in case of the UBDB construction (Volkov et al., 2004b; Dominiak et al., 2007; Jarzembska \& Dominiak, 2012) with some changes. The valence-only theoretical structure factors were computed (Frisch et al., 2009) from molecular wave functions obtained at the B3LYP/6-31G** (Becke, 1988; Perdew, 1986; Lee et al., 1988; Kendall et al., 1992) level of theory. The multipole model parameters were refined (Volkov et al., 2006b) against theoretical structure factors following various refinement strategies (see next subsection). The final averaging procedure of multipole model parameters over the atom types used to build UBDB was omitted. The multipole model parameters thus obtained constituted so-called tailored pseudoatoms, which means they were derived for and applied to the same molecules.

\section{1. 2. New models of electron densities from new refinement strategies}

From many possible strategies, only two of them lead to refinements stable enough to give promising electron density models: $\mathrm{P}_{\mathrm{v}} \kappa \mid \mathrm{P}_{\mathrm{Im}} \kappa^{\prime}$ and $\mathrm{P}_{\mathrm{v}} \kappa\left|\mathrm{P}_{\mathrm{Im}}\right| \kappa^{\prime}$.

In the first refinement strategy multipole model parameters were divided into two blocks: $P_{v}$ and $\kappa$ parameters describing valence electron densities, and $P_{l m}$ and $\kappa^{\prime}$ parameters describing angular deformations of electron densities. At the first step of the refinement, $P_{v}$ and $\kappa$ parameters were refined together for all atoms in the molecule, until the convergence was achieved. Then these 
parameters were fixed and the rest of the parameters, and $P_{l m}(l=1, \ldots, 4$ for non-hydrogen and $l=$ 1,2 for hydrogen atoms) and $\kappa^{\prime}$ (one for all levels of multipole expansion, from $l=1$ to $l=4$ ), were refined together until the convergence. The electron density model thus obtained we marked as $P_{\mathrm{v}} \kappa \mid P_{\operatorname{lm}} \kappa^{\prime}$.

Second strategy was similar, however, included three blocks of refined parameters and thus three stages of refinement. The first stage was the same, i.e. refinement of $P_{v}$ and $\kappa$ parameters. In the second stage, the values of $P_{v}$ and $\kappa$ parameters were kept fixed and only population parameters of multipole functions $P_{l m}(l=1, \ldots, 4$ for non-hydrogen and $l=1,2$ for hydrogen atoms) were refined. Finally the refinement of $\kappa^{\prime}$ parameters (one for all levels of multipole expansion, from $l=1$ to $l=4$ ) was done in the presence of fixed values of the other parameters. The electron density model thus obtained we marked as $\mathrm{P}_{\mathrm{v}} \kappa\left|\mathrm{P}_{\mathrm{Im}}\right| \kappa^{\prime}$.

\subsubsection{Estimation of electrostatic energy with new models of electron densities}

The electrostatic interaction energies were estimated using the EPMM method (Volkov et al., 2004a) and the XD2006 software (Volkov et al., 2006b) as described in our previous publications (Bojarowski et al., 2016; 2017; 2018). It is a hybrid method of exact potential (EP) integration at closer distance $(5 \AA)$ and simple sum of multipole moments interactions (MM) at larger distances. The software allows to use the EP or MM methods separately, and it is possible to estimate the electrostatic energy at any level of the multipolar expansion as described in our previous publication (Bojarowski et al., 2016; 2017; 2018).

\subsubsection{Reference values of electrostatic energy}

As an ultimate reference we used quantum mechanics values obtained at the DFTSAPT/B3LYP/aug-cc-pVTZ level (Bojarowski et al., 2018). To validate if new models are better in the electrostatic energy estimation than already known models we used the results obtained from tailored UBDB approach (simultaneous refinement of all multipole model parameters) computed at the B3LYP/6-31G** as described in the 2.1.1 section, and from the aug-PROmol approach taken from our previous publications (Bojarowski et al., 2016; 2018).

\subsection{Experimental data collection and analysis}

\subsubsection{Crystallization, data collection and processing, structure solution and the IAM refinements}

\subsubsection{Cyanuric acid}

$8 \mathrm{mg}$ of cyanuric acid powder was added to $1.5 \mathrm{~mL}$ of water : ethanol (1:1, v:v) mixture, and it was dissolved in a water bath at $56 \mathrm{C}$, stirred from time to time. The solutions were left to evaporate at room temperature. Crystals appeared as early as the next day. Also during the rapid evaporation, when the previously cooled solution was poured onto the petri dish, single crystals with well-formed walls were formed. Due to instability both on air and in oil, crystals were mounted on the capillary and put into nitrogen stream at $100 \mathrm{~K}$ immediately after being crystalized.

Single-crystal X-ray diffraction measurement was performed at $100 \mathrm{~K}$ on Agilent Technologies SuperNova four-circle diffractometer with molybdenum radiation source equipped with a lowtemperature nitrogen gas-flow device (Oxford Cryosystems Cryostream Plus). The determination of unit-cell parameters, integration of reflection intensities and data reduction, including 3D profile fitting, following profile size changes with incidence angle, numerical absorption correction based on Gaussian integration and empirical absorption correction implemented in SCALE3 ABSPACK scaling algorithm, were performed using CrysAlisPro 1.171.39.43d (CrysAlisPRO, Oxford Diffraction /Agilent Technologies UK Ltd, Yarnton, England). Finally, reflections were merged with the SORTAV program 
(Blessing, 1987, 1995, 1997). Final data collection and reduction parameters are summarized in Table 1.

The structure was solved in the Olex2 suite (Dolomanov et al., 2009) using the SHELXT (Sheldrick, 2015a) program with Intrinsic Phasing method applied. Refinement with independentatom model (IAM) was performed with the SHELXL program (Sheldrick, 2015b).

\subsubsection{Triptycene - X-ray diffraction experiment}

Single crystals of triptycene were obtained from Dr Tomasz Ratajczyk from Institute of Organic Chemistry, Polish Academy of Sciences, Poland.

Single crystal X-ray diffraction data set was collected using a Bruker AXS Kappa APEX II Ultra single-crystal diffractometer equipped with a CCD-type APEX II area detector, molybdenum TXS rotating anode (Mo Ka radiation, $\lambda=0.71073 \AA$ ), four-circle goniometer, multi-layer optics, and a lowtemperature nitrogen gas-flow device by Oxford Cryosystems (700 Series Cryostream). The determination of the unit cell parameters and the integration of raw diffraction images were performed with the APEX3 program package (Bruker AXS Inc., Madison, Wisconsin, USA.). The data set was corrected for Lorentz, polarization, and oblique incidence effects. The multi-scan absorption correction, frame-to-frame scaling and merging of reflections were carried out with the SORTAV program (Blessing, 1987, 1995, 1997). The collected data set exhibited high resolution limit of $(\sin \theta / \lambda)$ $\max =1.23 \AA^{-1}$. Final data collection and reduction parameters are summarized in Table 1.

The structure was solved using direct methods as implemented in the SHELXS program ((Sheldrick, 2008), and refined with the SHELXL program (Sheldrick, 2015b) within the independent atom model (IAM) approximation.

\subsubsection{Triptycene - neutron diffraction experiment}

Reflection data from single-crystal time-of-flight neutron diffraction experiment were taken from Sanjuan-Szklarz et al., 2016. The crystal structure (with anisotropic hydrogen atom displacement parameters) was then re-refined in the JANA package (Petříček et al., 2014). The extinction was successfully modelled by the Becker-Coppens approach (Becker \& Coppens, 1974b, a, 1975).

Selected parameters: $a=8.1019(13) \AA, b=8.1922(13) \AA, c=20.442(3) \AA$; total no. of reflections: 21802; no. of reflections with $I \geq 3 \sigma(I)$ : 21749; index ranges: $-16 \leq h \leq 6,-20 \leq k \leq 21$, $-56 \leq l \leq 52$; no. of parameters: 316; discrepancy factors: $R[F](I \geq 3 \sigma(I))=6.27 \%, w R[F](I \geq 3 \sigma(I))$ $=9.87 \%, R[F]$ (all data) $=6.29 \%, w R[F]$ (all data) $=9.89 \%$, goodness of fit $(S)(I \geq 3 \sigma(I))=4.39$; nuclear density largest extrema: $-2.09 /+4.37 \mathrm{fm} \cdot \AA^{-3}$; extinction parameter (type I Lorentzian isotropic): 76.3(12).

\subsubsection{Multipole model refinements}

\subsubsection{Cyanuric acid dihydrate}

Multipole model refinement on experimental data was performed in the WinXD2016 package (Volkov et al., 2016) with the use of the Hansen-Coppens multipolar model (Hansen \& Coppens, 1978). The initial atomic coordinates for all atoms, the anisotropic atomic displacement parameters for nonhydrogen atoms and the isotropic atomic displacement parameters for hydrogen atoms were taken from the IAM refinement. Local Cartesian coordinate systems, local symmetry constrains, and the initial population and contraction-expansion parameters of multipole model were defined by LSDB (Volkov, Li et al., 2004) combined with UBDB2011 (Jarzembska \& Dominiak, 2012). Each atom was assigned core and spherical valence scattering factors derived from the Su \& Coppens (1998) atomic 
wave functions. The $\mathrm{X}-\mathrm{H}$ bond lengths were constrained to neutron-normalized distances $\left(d_{\mathrm{O}-\mathrm{H}}=\right.$ $0.958 \AA, d_{\mathrm{N}-\mathrm{H}}=1.030 \AA$ ) (Allen \& Bruno, 2010). The values of the anisotropic atomic displacement parameters for the hydrogen atoms were estimated from the SHADE 3.0 server (Madsen, 2006). For non-hydrogen atoms anisotropic atomic displacement parameters were refined in harmonic approximation, except $\mathrm{O} 2$ and $\mathrm{O} 3$ oxygen atoms, for which anharmonic approximation was applied. Gram-Charlier coefficients (Johnson, 1969; Kuhs, 1983; Scheringer, 1985) up to the fourth order were used, while the physical reliability of the anharmonic model was confirmed by the probability density function computed with XDPDF from the WinXD2016 suite. The multipole expansion was truncated at the hexadecapole $\left(l_{\max }=4\right)$ and quadrupole $\left(l_{\max }=2\right)$ levels for all non-hydrogen and hydrogen atoms, respectively. Only parameters allowed by local and crystallographic symmetry were refined. $\mathrm{k}$ and $\mathrm{K}^{\prime}$ parameters for hydrogen atoms were kept fixed at the UBDB-transferred values. The general multipole model refinement strategy consisted of the following steps: the refinement of (i) scale factor (which was also refined in all other stages); (ii) atomic coordinates and ADPs for all atoms; (iii) introduction of $\mathrm{X}-\mathrm{H}$ constrains, refinement of atomic coordinates and ADPs for non-hydrogen atoms; (iv) SHADE estimation of anisotropic hydrogen atom ADPs; $(v)$ atomic coordinates and ADPs refinement for nonhydrogen atoms including the $3^{\text {rd }}$ and $4^{\text {th }}$ order anharmonic parameters for the $\mathrm{O} 2$ and $\mathrm{O} 3$ oxygen atoms; (vi) atomic coordinates and ADPs for non-hydrogen atoms, multipole population and expansion-contraction parameters in a stepwise manner; (vii) update of SHADE estimation of anisotropic hydrogen atom ADPs; (viii) all parameters simultaneously.

Refinements were carried out against $F^{2}$ on all data up to $\sin \theta / \lambda \leq 1.33 \AA^{-1}$, without any $F^{2} / \sigma\left(F^{2}\right)$ cut-off, using statistical weights (i.e. for $i$-th reflection $w_{i}=1 / \sigma\left(F^{2}\right)_{i}^{2}$ ).

Correctness of the multipole model refinement was judged from refinement statistics $(R[F]$, $w R 2\left[F^{2}\right]$ and goodness of fit $\left.S\left[F^{2}\right]\right)$, differences of mean-square displacement amplitudes (DMSDA) for non-hydrogen atoms, and residual density analysis. The largest DMSDA value of $-4 \cdot 10^{-4} \AA^{2}$ was observed for the $\mathrm{C} 2=\mathrm{O} 2$ bond, which is satisfactory. Comparisons of $F_{o b s}^{2}$ and $F_{o b s}^{2}$ (Figure 1S) shows that they scale to each other with the same proportion over all resolution bins and the differences between them (intensity residuals) have close to normal distribution. Residual electron density analysis (Meindl \& Henn, 2008) shows that the derived model is characterized by rather flat and featureless residual density distribution (Figures 2S, 4S).

All the final refinement statistics are summarized in Table 1. The ORTEP plot for the asymmetric unit is given in Figure 1.

\subsubsection{Triptycene}

Multipole model refinement was carried out using the MoPro suite (Guillot et al., 2001; Jelsch et al., 2005) with the use of the Hansen-Coppens multipole model (Hansen \& Coppens, 1978). The initial atomic coordinates for all atoms, the anisotropic atomic displacement parameters for nonhydrogen atoms and the isotropic atomic displacement parameters for hydrogen atoms were taken from the IAM refinement. Local Cartesian coordinate systems, local symmetry constrains and the initial population and contraction-expansion parameters of multipole model were defined by LSDB (Volkov, Li et al., 2004) combined with UBDB2011 (Jarzembska \& Dominiak, 2012). Each atom was assigned core and spherical valence scattering factors derived from the Su \& Coppens (1998) atomic wave functions. The $\mathrm{X}-\mathrm{H}$ bond lengths were restrained with $\sigma=0.001 \AA$ to distances derived from neutron diffraction data. The values of the anisotropic atomic displacement parameters for the hydrogen atoms were transferred from the neutron diffraction data with appropriate scale. For nonhydrogen atoms anisotropic atomic displacement parameters (anisotropic ADPs) were refined in harmonic approximation. The multipole expansion was truncated at the hexadecapole $\left(l_{\max }=4\right)$ and 
quadrupole $\left(l_{\max }=2\right)$ levels for all non-hydrogen and hydrogen atoms, respectively. Only parameters allowed by local and crystallographic symmetry were refined. Constrains resulting from chemical similarities were applied to multipole model parameters. $\mathrm{k}$ parameters for hydrogen and $\mathrm{k}^{\prime}$ parameters for all atoms were kept fixed at the UBDB-transferred values. The general multipole model refinement strategy consisted of the following steps: the refinement of (i) scale factor (which was also refined in all other stages); (ii) atomic coordinates; (iii) atomic coordinates and ADPs; (iv) SHADE estimation of anisotropic hydrogen atom ADPs (which was also updated in-between other stages until convergence); (v) multipole population parameters in a stepwise manner; (vi) all multipole population parameters and structural parameters simultaneously; (vii) block refinement of non-hydrogen atom $\kappa$ parameters ( $1^{\text {st }}$ block), step no. (vi) ( $2^{\text {nd }}$ block); (viii) all parameters from step no (vii) together; (ix) anisotropic hydrogen ADPs transfer from neutron diffraction data; $(x)$ all parameters simultaneously.

Refinements were carried out against $F^{2}$ on all data up to $\sin \theta / \lambda \leq 1.23 \AA^{-1}$, without any $F^{2} / \sigma\left(F^{2}\right)$ cut-off, using statistical weights (i.e. for $i$-th reflection $\left.w_{i}=1 / \sigma\left(F^{2}\right)_{i}^{2}\right)$.

Correctness of the multipole model refinement was judged from refinement statistics $(R[F]$, $w R 2\left[F^{2}\right]$ and goodness of fit $\left.S\left[F^{2}\right]\right)$, differences of mean-square displacement amplitudes (DMSDA) for non-hydrogen atoms, and residual density analysis. The largest DMSDA value of $-5 \cdot 10^{-4} \AA^{2}$ was observed for the C1-C15 and C2-C16 bonds, which is satisfactory. Comparisons of $F_{o b s}^{2}$ and $F_{o b s}^{2}$ (Figure 1S) shows that they scale to each other with the same proportion over all resolution bins and the differences between them (intensity residuals) have close to normal distribution. Residual electron density analysis (Meindl \& Henn, 2008) shows that the derived model is characterized by rather flat and featureless residual density distribution (Figures 3S, 4S).

All the final refinement statistics are summarized in Table 1. The ORTEP plot for the asymmetric unit is given in Figure 1.
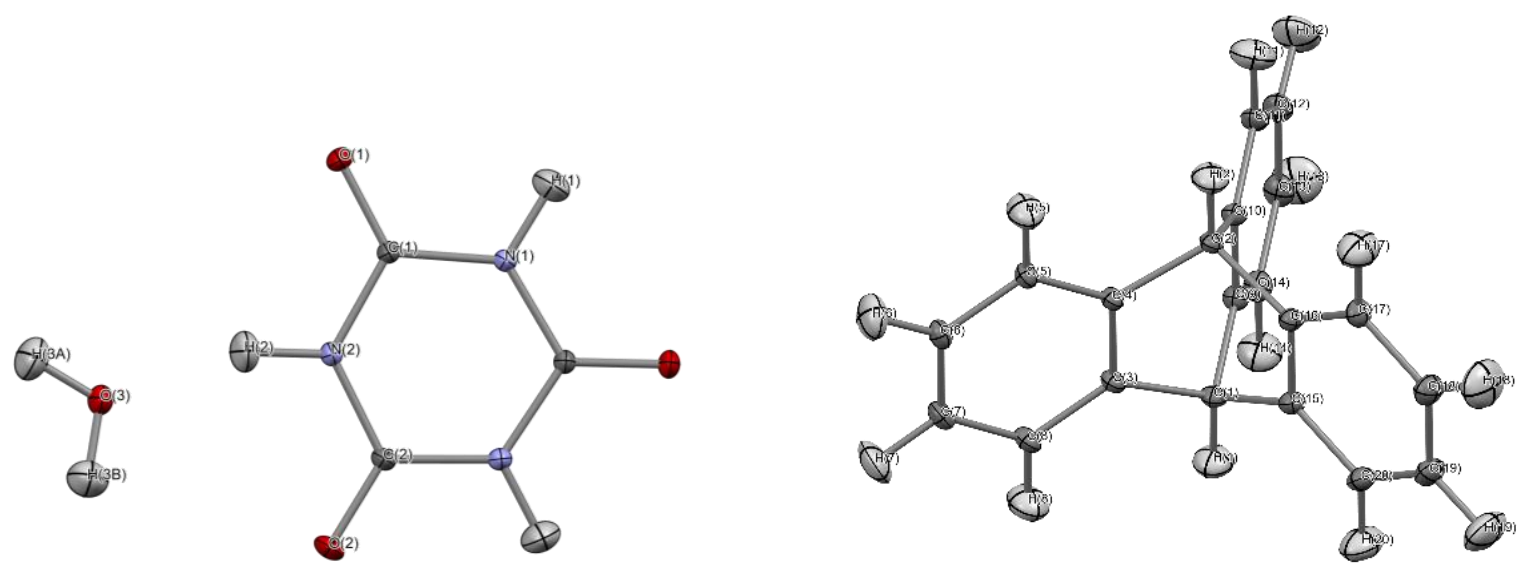

Figure 1. ORTEP plot of for cyanuric acid dihydrate (left) and triptycene (right) after multipole model refinement on experimental X-ray data drawn with Mercury (Macrae et al., 2008). Ellipsoids drawn at $50 \%$. 
Table 1. Parameters characterizing the studied crystals, diffraction data collection and refinement.

\begin{tabular}{|c|c|c|}
\hline \multicolumn{3}{|l|}{ Crystal parameters } \\
\hline Name & $\begin{array}{l}\text { Cyanuric acid } \\
\text { dihydrate }\end{array}$ & Triptycene \\
\hline Formula & $\mathrm{C}_{3} \mathrm{H}_{3} \mathrm{~N}_{3} \mathrm{O}_{3}, 2\left(\mathrm{H}_{2} \mathrm{O}\right)$ & $\mathrm{C}_{20} \mathrm{H}_{14}$ \\
\hline $\begin{array}{l}\text { Formula weight, } \\
M_{\mathrm{r}} / \mathrm{g} \cdot \mathrm{mol}^{-1}\end{array}$ & 165.12 & 254.31 \\
\hline Space group & $I 2 / a($ no. 15$)$ & $P 2_{1} 2_{1} 2_{1}$ (no. 19) \\
\hline$Z$ & 4 & 4 \\
\hline$F_{000}$ & 344 & 536 \\
\hline $\begin{array}{l}\text { Crystal color } \\
\text { and habit }\end{array}$ & colorless, block & colorless, block \\
\hline Crystal size & $0.31 \times 0.23 \times 0.18$ & $0.10 \times 0.16 \times 0.34$ \\
\hline \multicolumn{3}{|l|}{ Diffraction data parameters } \\
\hline Temperature, $T$ / K & $100 \mathrm{~K}$ & $100 \mathrm{~K}$ \\
\hline$a / \AA$ & $8.6385(1)$ & $8.0808(3)$ \\
\hline$b / \AA ̊$ & 6.6817 & $8.1662(3)$ \\
\hline$c / \AA$ & $11.6069(1)$ & $20.3821(8)$ \\
\hline$\beta /{ }^{\circ}$ & $107.466(1)$ & 90 \\
\hline Volume, $V / \AA^{3}$ & $639.061(10)$ & $1345.00(9)$ \\
\hline$d_{\text {calc }} / \mathrm{g} \cdot \mathrm{cm}^{-3}$ & 1.716 & 1.256 \\
\hline Absorption coefficient, $\mu / \mathrm{mm}^{-1}$ & 0.163 & 0.071 \\
\hline$\theta$ range & $3.505^{\circ}-71.095^{\circ}$ & $2.69^{\circ}-60.68^{\circ}$ \\
\hline \multirow{2}{*}{$(\sin \theta / \lambda)_{\max } / \AA^{-1}$} & 1.33 & 1.23 \\
\hline & $-22 \leq h \leq 22$ & $0 \leq h \leq 19$ \\
\hline \multirow[t]{2}{*}{ Index ranges } & $-17 \leq k \leq 17$ & $0 \leq k \leq 20$ \\
\hline & $-30 \leq l \leq 30$ & $-49 \leq l \leq 49$ \\
\hline $\begin{array}{l}\text { No. of reflections } \\
\text { collected / unique }\end{array}$ & 116909 / 6322 & $110051 / 20485$ \\
\hline Completeness & $99.4 \%$ & $98.5 \%$ \\
\hline$R_{\mathrm{mrg}}$ ๆ & $2.55 \%$ & $3.89 \%$ \\
\hline $\begin{array}{l}\text { No. of reflections } \\
\text { with } I \geq 3 \sigma(I)\end{array}$ & $5349(84.1 \%)^{\$}$ & $16190(77.8 \%)^{\$}$ \\
\hline \multicolumn{3}{|l|}{ Refinement parameters $^{\#}$} \\
\hline $\begin{array}{l}\text { No. of reflections / parameters } \\
\text { / restraints }\end{array}$ & 6322 /198 / 0 & 20485 / 263 / 14 \\
\hline$R[F](I \geq 3 \sigma(I)) /$ (all data) & $1.15 \% / 1.72 \%$ & $1.95 \% / 3.01 \%$ \\
\hline$w R 2\left[F^{2}\right](I \geq 3 \sigma(I)) /$ (all data) & $3.18 \%$ / 3.41\% & $4.25 \% / 4.62 \%$ \\
\hline$R\left[F^{2}\right](I \geq 3 \sigma(I)) /($ all data $)$ & $1.59 \% / 1.62 \%$ & $2.37 \% / 2.59 \%$ \\
\hline$S\left[F^{2}\right](I \geq 3 \sigma(I)) /($ all data $)$ & $0.989 / 0.979$ & $0.965 / 0.933$ \\
\hline$\Delta \rho_{\min } / \Delta \rho_{\max } / \mathrm{e} \cdot \AA^{-3}(I \geq 3 \sigma(I))$ & $-0.14 /+0.15$ & $-0.14 /+0.17$ \\
\hline$\Delta \rho_{\min } / \Delta \rho_{\max } / \mathrm{e} \cdot \AA^{-3}$ (all data) & $-0.15 /+0.14$ & $-0.20 /+0.19$ \\
\hline \multicolumn{3}{|c|}{$\begin{array}{l}\text { " Definition of } R_{\mathrm{mrg}} \text { follows Blessing's definitions used in the SORTAV program: } \\
R_{m r g}=\sum_{h k l} \sqrt{n /(n-1)} \sum_{i=1}^{n}\left|I_{i}-I_{\text {mean }}\right| / \sum_{h k l} \sum_{i=1}^{n} I_{i} .{ }^{\$} \text { Numbers in brackets } \\
\text { constitute the percentage of the 'observed' unique reflections with respect to } \\
\text { the total number of unique reflections. }{ }^{\#} \text { In the case of X-ray diffraction from } \\
\text { multipole model refinements. }\end{array}$} \\
\hline
\end{tabular}




\subsubsection{TAAM refinements}

TAAM refinements were carried out on $F^{2}$, without any $F^{2} / \sigma\left(F^{2}\right)$ cut-off, using statistical weights (i.e. for $i$-th reflection $w_{i}=1 / \sigma\left(F^{2}\right)_{i}^{2}$ ) in the XD2016 package. Dataset of two resolutions were used: truncated at atomic resolution $d_{\min }=0.83 \AA\left(\sin \theta / \lambda \leq 0.60 \AA^{-1}\right)$, and of sub-atomic highresolution resulting from the experiment - the same as for multipole model refinement. Atomic coordinates and atomic displacement parameters (anisotropic for non-hydrogen and isotropic for hydrogen atoms) for all atoms were simultaneously refined with no restrains. In addition, for $\mathrm{O} 2$ and $\mathrm{O} 3$ oxygen atoms in cyanuric acid dihydrate anharmonic approximation was used with Gram-Charlier coefficients (Kuhs, 1983; Johnson, 1969; Scheringer, 1985) up to the fourth order. The number of unique reflections at atomic resolution and of refined parameters in TAAM refinements for cyanuric acid dihydrate was 581 and 104, respectively, and for triptycene 2427 and 237, respectively.

TAAM was parametrized with tailored UBDB approach, $\mathrm{P}_{\mathrm{v}} \kappa \mid \mathrm{P}_{\mathrm{Im}} \kappa^{\prime}$ and $\mathrm{P}_{\mathrm{v}} \kappa\left|\mathrm{P}_{\mathrm{Im}}\right| \kappa^{\prime}$ models, following procedures as described in 2.1.2 on the basis of molecular geometries taken from multipole models refined on experimental data. For refinements on low resolution data, TAAM parametrized with multipole model parameters transferred from multipole model refinements on high-resolution data (MMR) was also tested. In addition, aug-PROmol model was constructed on the same geometries following procedure described in (Bojarowski et al., 2016).

\section{Results and discussion}

\subsection{Refinement strategies}

Following the arguments mentioned in the introductory section, one can imagine several possible refinement strategies to obtain new models of electron densities. The most strict cumulative approach would be to refine each level of multipole expansion separately, one after another, i.e. refining higher multipole parameters in the presence of previously refined and frozen parameters of lower multipoles. With Hansen-Coppens multipole model that would be the following consecutive steps: $P_{v}$ and $\kappa, P_{1 m}$ and $\kappa_{1}^{\prime}, P_{2 m}$ and $\kappa_{2}^{\prime}, P_{3 m}$ and $\kappa_{3}^{\prime}$ and $P_{4 m}$ and $\kappa_{4}^{\prime}$. One can be even more restrictive, and at each level of multipole expansion refine firstly population parameters, then froze them and refine kappa parameters of that level. These two strategies turned out to be very unstable during refinement of Hansen-Coppens multipole model. There were often problems with achieving refinement convergence, or values of refined parameters were far from the acceptable (i.e. $\kappa$ and $\kappa^{\prime}$ parameters much different from one). Only two strategies lead to stable refinements and promising electron density models: $\mathrm{P}_{\mathrm{v}} \kappa \mid \mathrm{P}_{\mathrm{lm}} \kappa^{\prime}$ model were $P_{v}$ and $\kappa$ parameters were refined firstly, then $P_{l m}(l=$ $1, \ldots, 4$ ) and $\kappa^{\prime}$ (as one parameter for all levels of multipole expansion, $\kappa^{\prime}=\kappa_{1}^{\prime}+\kappa_{2}^{\prime}+\kappa_{3}^{\prime}+\kappa_{4}^{\prime}$ ); and $\mathrm{P}_{\mathrm{v}} \kappa\left|\mathrm{P}_{\mathrm{Im}}\right| \kappa^{\prime}$ model were the second stage was divided into two: firstly $P_{l m}$, then $\kappa^{\prime}$ parameters.

\subsection{Electrostatic energy estimations from new electron density models}

The first model combined with the EPMM method of electrostatic energy calculation, $\mathrm{P}_{\mathrm{v}} \kappa \mid \mathrm{P}_{\mathrm{Im}} \kappa^{\prime}+\mathrm{EPMM}$, always showed a similar trend in computed electrostatic energy values as tailored UBDB+EPMM and REF (Figure 2). This is intuitive, as the refinement procedure is the closest to that of UBDB. Nevertheless, RMSE for the $\mathrm{P}_{\mathrm{v}} \kappa \mid \mathrm{P}_{\mathrm{Im}} \kappa^{\prime}+$ EPMM method is larger, by up to ca $50 \%$, in almost all analyzed subgroups of $566 \times 8$ dataset comparing to the UBDB+EPMM.

When comparing the two tested models, the most important conclusion seems to be the enormous influence of the $\kappa^{\prime}$ value on the accuracy of the electrostatic energy estimation. The $\mathrm{P}_{\mathrm{v}} \kappa\left|\mathrm{P}_{\mathrm{Im}}\right| \kappa^{\prime}$ model generates electrostatic energies which are much far away from the reference values, 
with RMSE twice or even more larger than for the first model. Apparently population and expansioncontraction parameters are so strongly related with each other that they must be refined simultaneously (or at least iteratively, not cumulatively).

The second important finding is the overestimation of electrostatic energy. While the main reason for the need to develop further UBDB was the underestimation of the energy, the new models overestimate electrostatic interactions.

Interestingly, when comparing the energies obtained from models truncated to the monopole level $(l=0)$, a significant improvement in the estimation of electrostatic energy is seen with new model(s) over the tailored UBDB at $l=0$. Please note that both new models when truncated to monopole level simplify to one the same model, since now marked as $\mathrm{P}_{\mathrm{v}} \kappa$. With application of the MM method of calculations, the $\mathrm{P}_{\mathrm{v}} \kappa$ model already at $l=0$ gives results very close to the results of the RESP charges. At the same time UBDB truncated at $l=0$ is much worse and only at $l=4$ approaches similar level of accuracy. When the EPMM method is used, the results are slightly inferior for the PVK model, but still promising. The $\mathrm{P}_{\mathrm{v}} \kappa$ model is significantly worse only for short distances in electrostatic dimers. Knowing the construction of the aug-PROmol model (Bojarowski et al., 2018), it can be concluded that expansion-contraction parameters are very important and their fine tuning may lead in the future to even better results.
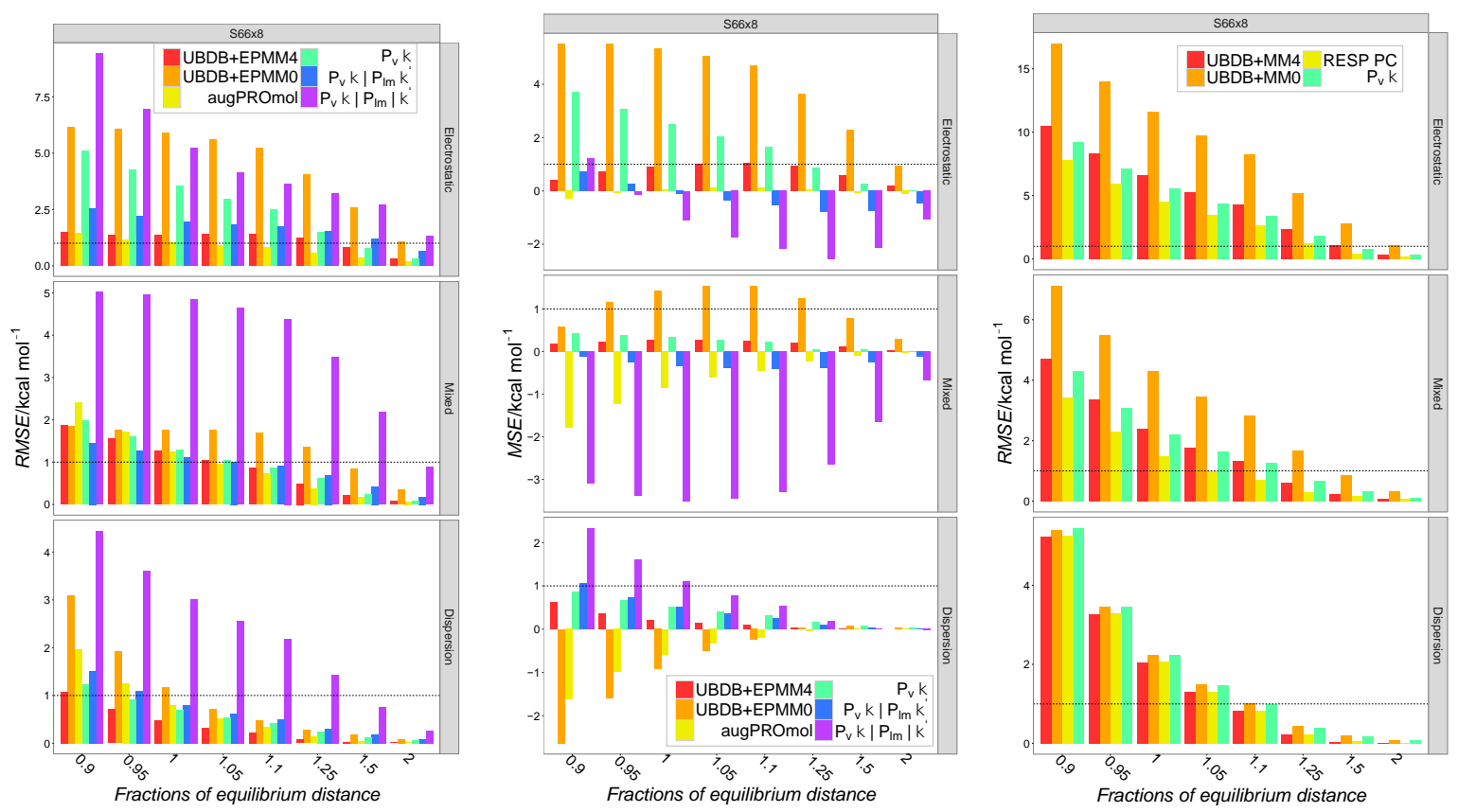

Figure 2. Visualization of the Root Mean Square Error (RMSE) and the Mean Signed Error (MSE) of the electrostatic interaction energies $\left(\mathrm{kcal}^{\mathrm{mol}}{ }^{-1}\right)$ computed with the new models $\left(\mathrm{P}_{\mathrm{v}} \kappa\left|\mathrm{P}_{\mathrm{Im}} \kappa^{\prime}, \mathrm{P}_{\mathrm{v}} \kappa\right| \mathrm{P}_{\mathrm{Im}} \mid \kappa^{\prime}\right.$ and $\left.\mathrm{P}_{\mathrm{v}} \kappa\right)$ at different intermolecular distances in three subgroups of the $\mathrm{S} 66 \times 8$ datasets. Results from other approaches (tailored UBDB and aug-PROmol) are given to allow direct comparisons. The energies were computed by applying the EPMM or the MM methods, either to full models, up to $l=4$ (EPMM4, $\mathrm{MM} 4)$ or truncated at $l=0$ (EPMMO, MMO). Corresponding reference energies were obtained at DFTSAPT/B3LYP/aug-cc-pVTZ level of theory. The dashed horizontal lines along the $x$ axis correspond to the value of $1.0 \mathrm{kcal} \mathrm{mol}^{-1}$.

\subsection{Crystal structure refinements on experimental X-ray diffraction data}

To validate the performance of tested models as a source of better atomic X-ray scattering factors we focused on two aspects of X-ray structure refinement: (a) how well the model can be fitted 
to experimental data, and (b) how accurate structures can be obtained with the model. In addition, we tested the models in two situations, firstly in refinements against sub-atomic high-resolution data, secondly in standard type of refinements with atomic resolution of data $\left(d_{\min }=0.83 \AA\right.$ ). As an ultimate reference we used results from multipole model refinements (MMR) on high-resolution data, and in case of triptycene, from neutron diffraction. We also had several TAAM refinement reference points. Firstly, we did TAAM refinements with tailored UBDB parametrization, TAAM(UBDB). Secondly, we did TAAM refinements with multipole model parameters taken from multipole model refinements, TAAM(MMR).

To check the quality of the fitting we focused on the following fitting statistics: $R[F], w R 2\left[F^{2}\right]$ and maximum and minimum residual peaks, see Table 2. From all fitting statistics it is clear that tested models, $\mathrm{P}_{\mathrm{v}} \kappa \mid \mathrm{P}_{\mathrm{Im}} \kappa^{\prime}$ and $\mathrm{P}_{\mathrm{v}} \kappa\left|\mathrm{P}_{\mathrm{Im}}\right| \kappa^{\prime}$, are able to fit the experimental data much better than standard IAM. This is true for both, standard atomic and subatomic high-resolution datasets. $R[F]$ lowers by ca. 1 percent point, and reduction in absolute values of extrema for residual electron densities is observed. From comparing values of fitting statistics for $\mathrm{P}_{\mathrm{v}} \kappa \mid \mathrm{P}_{\mathrm{Im}} \kappa^{\prime}$ and $\mathrm{P}_{\mathrm{v}} \kappa\left|\mathrm{P}_{\mathrm{Im}}\right| \kappa^{\prime}$, it can be concluded that the first model fits the data slightly better, but the difference is very small. None of the tested models, however, fits the data as good as tailored UBDB model. UBDB allows for further lowering of the R(F) factor, by additional ca. 0.5 percent point, and further lowering of residual extrema, especially in case of cyanuric acid dihydrate. In fact, tailored UBDB model fits to the data almost as good as refined multipole model (MMR), the latter gives clearly better statistics only in case of refinements on highresolution data for cyanuric acid dihydrate. It is worth to remember that with full multipole model refinements, much larger number of parameters is fitted to the data than in the case of TAAM or IAM refinements. The aug-PROmol model, which was very successful in electrostatic interaction energy estimations, performs very badly in X-ray crystal structure refinements. It is able to fit to the experimental data at the level similar to the standard IAM (for triptycene), or even slightly worse (for cyanuric acid dihydrate).

Table 2. Fitting statistics computed for all reflections from refinements on experimental X-ray diffraction data with the usage of various electron density models.

\begin{tabular}{|c|c|c|c|c|c|c|c|c|}
\hline & \multicolumn{4}{|c|}{ cyanuric acid dihydrate } & \multicolumn{4}{|c|}{ triptycene } \\
\hline & $\begin{array}{c}R[F] \\
\%\end{array}$ & $\begin{array}{c}w R 2\left[F^{2}\right] \\
\%\end{array}$ & $\begin{array}{c}\Delta \rho_{\min } \\
\mathrm{e} \cdot \AA^{-3}\end{array}$ & $\begin{array}{c}\Delta \rho_{\max } \\
\mathrm{e} \cdot \AA^{-3}\end{array}$ & $\begin{array}{c}R[F] \\
\%\end{array}$ & $\begin{array}{c}w R 2\left[F^{2}\right] \\
\%\end{array}$ & $\begin{array}{c}\Delta \rho_{\min } \\
\mathrm{e} \cdot \AA^{-3}\end{array}$ & $\begin{array}{c}\Delta \rho_{\max } \\
\mathrm{e} \cdot \AA^{-3}\end{array}$ \\
\hline \multicolumn{9}{|l|}{ Atomic resolution } \\
\hline IAM $^{\pi}$ & 2.7 & 9.6 & -0.25 & 0.21 & 2.7 & 7.3 & -0.20 & 0.12 \\
\hline aug-PROmol & 3.1 & 9.1 & -0.37 & 0.28 & 2.7 & 7.5 & -0.19 & 0.13 \\
\hline $\operatorname{TAAM}\left(\mathrm{P}_{\mathrm{v}} \kappa \mid \mathrm{P}_{\operatorname{lm}} \kappa^{\prime}\right)$ & 1.5 & 4.8 & -0.17 & 0.11 & 1.4 & 3.8 & -0.09 & 0.09 \\
\hline $\operatorname{TAAM}\left(\mathrm{P}_{\mathrm{v}} \kappa\left|\mathrm{P}_{\operatorname{lm}}\right| \kappa^{\prime}\right)$ & 1.6 & 5.4 & -0.16 & 0.14 & 1.4 & 3.9 & -0.09 & 0.09 \\
\hline TAAM(UBDB) & 0.9 & 2.8 & -0.08 & 0.07 & 1.1 & 3.2 & -0.09 & 0.07 \\
\hline TAAM(MMR) & 0.8 & 2.4 & -0.07 & 0.05 & 1.1 & 3.3 & -0.08 & 0.07 \\
\hline \multicolumn{9}{|c|}{ Subatomic resolution } \\
\hline IAM $^{9}$ & 3.0 & 8.1 & -0.33 & 0.65 & 4.2 & 8.2 & -0.25 & 0.50 \\
\hline aug-PROmol & 3.4 & 9.5 & -0.47 & 0.83 & 4.2 & 8.2 & -0.28 & 0.51 \\
\hline TAAM(Pv $\left.\operatorname{P} \mid \mathrm{P}_{\operatorname{lm}} \kappa^{\prime}\right)$ & 2.3 & 5.6 & -0.29 & 0.39 & 3.4 & 5.3 & -0.21 & 0.28 \\
\hline $\operatorname{TAAM}\left(\mathrm{P}_{\mathrm{v}} \kappa\left|\mathrm{P}_{\mathrm{Im}}\right| \kappa^{\prime}\right)$ & 2.6 & 6.7 & -0.30 & 0.58 & 3.4 & 5.4 & -0.22 & 0.27 \\
\hline TAAM(UBDB) & 1.9 & 4.1 & -0.23 & 0.21 & 3.1 & 4.7 & -0.20 & 0.22 \\
\hline
\end{tabular}


Besides the fitting statistics, it is important to validate the quality of obtained structural model. In case of X-ray diffraction data, the most sensitive parameters are the $\mathrm{X}-\mathrm{H}$ bond lengths and atomic displacement parameters. With cyanuric acid dihydrate structure we have in addition an unique opportunity to observe the performance of various models in deconvolution of static electron density from anharmonic motion of atoms. To compare the performance in atomic displacement modeling we focused on analysis of Ueq for non-H atoms and Uiso for hydrogen atoms, as well as on averaged absolute values of DMSDA analysis for bonds between non- $\mathrm{H}$ atoms. Following our previous experience on sensitivity of atomic displacement parameters to used electron density models and to data resolution, we focused only on atomic resolution refinements, since these are by far more responsive than refinements on subatomic resolution data (Sanjuan-Szklarz et al., 2020).

Surprisingly, it not so clear anymore if the tested new models are better or not than the IAM in obtaining more accurate crystal structures, Table 3. Positions of hydrogen atoms do improve with new models. RMSD for the $\mathrm{X}-\mathrm{H}$ bond lengths are in the range of $0.02-0.04 \AA$ when compared to referential mean values from neutron diffraction (Allen \& Bruno, 2010) or neutron diffraction data collected directly for triptycene. In case of IAM the RMSD was $0.14 \AA$ and $0.10 \AA$ for cyanuric acid dihydrate and triptycene, respectively. The improvement with new models is of the same level as for the tailored UBDB approach. It is only slightly worse than from TAAM (MMR) refinement, which arrived at RMSD of $0.01 \AA$. Note that MMR was constrained or restrained to neutron data. On the other hand, both new models have some problems in refinement of atomic displacement parameters. The first model, $P_{v} \kappa \mid P_{I m} \kappa^{\prime}$, is visibly better than IAM and as good as UBDB only in the case of triptycene. For triptycene the RMSD of Ueq of non-hydrogen atoms and of Uiso for hydrogen gets smaller by ca. three times comparing to referential neutron diffraction data. DMSDA improves as well. However, for cyanuric acid dihydrate the $\mathrm{P}_{\mathrm{v}} \kappa \mid \mathrm{P}_{\mathrm{Im}} \kappa^{\prime}$ model is slightly worse in Uiso refinement of hydrogen atoms than IAM, and for non-hydrogen atoms is somewhere in between IAM and UBDB in quality of Ueq but is worse than IAM in refinement of atomic displacement anisotropy. The second model, $\mathrm{P}_{\mathrm{v}} \kappa\left|\mathrm{P}_{\mathrm{Im}}\right| \kappa^{\prime}$, performs even worse than the first one, even for triptycene. It is especially visible for non-hydrogen atoms and for refining correct anisotropy of non-hydrogen atoms

Table 3. Statistics for selected structural parameters from refinements on experimental X-ray diffraction data with the usage of various electron density models.

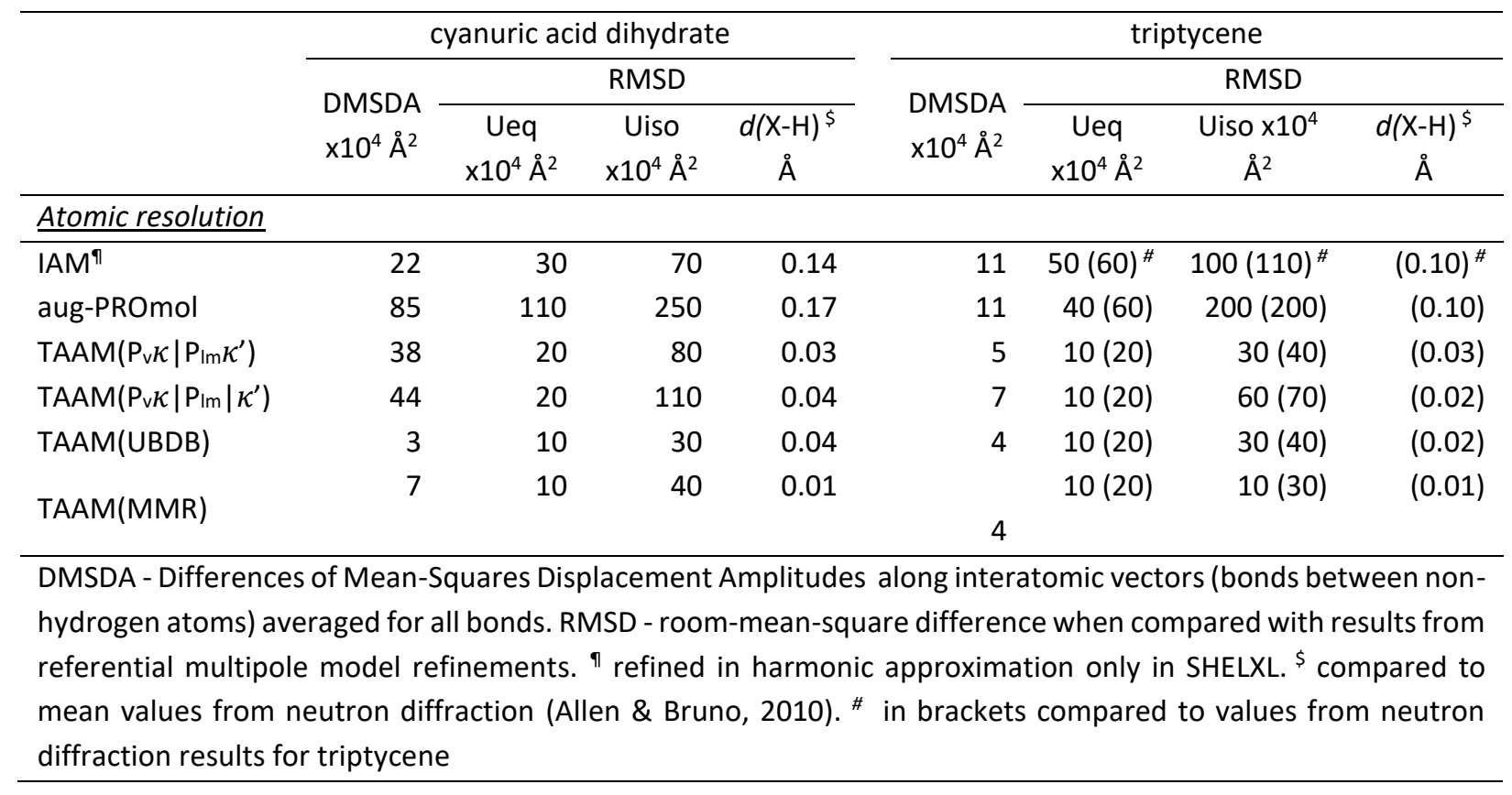


The aug-PROmol model performs the worst. It does not improve positions of hydrogen atoms, it makes them even worse than IAM in case of cyanuric acid dihydrate. Similarly, it does not improve atomic displacement parameters, or makes them even worse. The model acts especially badly in refinement of atomic displacements in the presence of anharmonic motion.

\section{Conclusions}

Cumulative approach to multipole model refinements, as oppose to simultaneous refinement of all multipole model parameters, leads to substantially different models of electron density.

From the two tested models, resulting from two slightly different strategies of cumulative refinement of multipole model, the second one, $\mathrm{P}_{\mathrm{v}} \kappa\left|\mathrm{P}_{\mathrm{Im}}\right| \kappa^{\prime}$, is of no use neither in electrostatic interaction energy estimations nor in X-ray crystal structure refinements. In the latter area of applications the consequences of improper treatment of $\kappa^{\prime}$ parameters has the largest consequences in modelling atomic displacement parameters. Thus it might be concluded that cumulative refinement of $P_{l m}$ and $\kappa^{\prime}$ parameters of multipole model is much worse than simultaneous. Therefore such an approach to the multipole model refinement should never be used and whenever simultaneous refinement of $P_{l m}$ and $\kappa^{\prime}$ is not stable, care must be taken to assure that iterative refinement converged.

The first tested model, $P_{\mathrm{v}} \kappa \mid P_{\operatorname{lm}} \kappa^{\prime}$, resulting from cumulative refinement of two blocks of parameters, $P_{v}$ and $\kappa$ and $P_{l m}$ and $\kappa^{\prime}$, have promising properties. It models quite well non-polar molecules or chemical fragments. For non-polar molecule (triptycene) it was better than IAM and only slightly worse than UBDB in X-ray crystal structure refinement. For molecular dimers in which dispersive intermolecular interaction dominates, or are similarly important as electrostatic interactions, it estimates electrostatic interaction energies computed with the EPMM approach similarly well as UBDB, and at short distance better than aug-PROmol. For polar molecules, however, the $\mathrm{P}_{\mathrm{v}} \kappa \mid \mathrm{P}_{\mathrm{Im}} \kappa^{\prime}$ model have some problems with proper refinement of atomic displacement parameters (probably enhanced by the presence of anharmonic motion) and is slightly worse than UBDB in estimation of electrostatic energy.

The $\mathrm{P}_{\mathrm{v}} \kappa \mid \mathrm{P}_{\mathrm{Im}} \kappa^{\prime}$ model have interesting properties when truncated to the monopole level - the $\mathrm{P}_{\mathrm{v}} \kappa$ model. The charges derived from the $\mathrm{P}_{\mathrm{v}} \kappa$ model behave similarly to the RESP charges in electrostatic interaction energy estimations from point charges (the MM method). In this respect the $\mathrm{P}_{\mathrm{v}} \kappa$ model is much better than the UBDB model which only at full expansion, up to octupole level to be more precise as we shown in Bojarowski et al. (2017), approaches the quality of energy estimations which have the RESP charges. This indirectly shows than electrostatic potential at the molecular surface computed from the point charges derived from the $P_{v} \kappa$ model is already very good. The $P_{v} \kappa$ model is also on promising model to replace the aug-PROmol approach. When combined with the EPMM method of electrostatic energy calculations it is as good as the aug-PROmol for mixed dimers, a bit better for dispersion-dominated dimers and worse only for electrostatic-dominated dimers. Apparently, some improvements in $\kappa$ parametrization for polar groups is still needed.

In summary it can be concluded that the $\mathrm{P}_{\mathrm{v}} \kappa \mid \mathrm{P}_{\mathrm{Im}} \kappa^{\prime}$ model is very promising and after some improvements it has a chance to be not worse than UBDB in X-ray crystal structure refinements and at the same moment, with the same route of model parameterization, it may deliver the $\mathrm{P}_{\mathrm{v}} \kappa$ model not worse than the aug-PROmol model in speed and accuracy of electrostatic energy estimation.

In addition, we showed that the aug-PROmol model, although is still at the moment the best spherical model for electrostatic interaction energy estimations, is not suitable for crystal X-ray 
structure refinement. It neither fits to the X-ray data nor leads to structural model better than IAM. It may be even worse than IAM in some cases.

Acknowledgements

The financial support to $S A B$ from the National Science Centre, Poland, grant No. $2015 / 19 /$ N/ST4/00010 is acknowledged. The access to the computational resources provided by the PL-GRID Infrastructure, grants No. ubdb2018 and ubdb2019, is acknowledged. We also thank dr Anna Makal, University of Warsaw, Poland and Oxford Diffraction division of Rigaku, Poland for help in preliminary high-resolution X-ray diffraction measurements for cyanuric acid dihydrate; and dr Tomasz Ratajczyk, Polish Academy of Sciences, Poland for providing crystals of triptycene.

\section{References}

Allen, F. H. \& Bruno, I. J. (2010). Acta Cryst. B66, 380-386.

Bąk, J. M., Domagała, S., Hübschle, C., Jelsch, C., Dittrich, B. \& Dominiak, P. M. (2011). Acta Cryst. A67, 141-153.

Becke, A. D. (1988). Phys. Rev. A, 38, 3098-3100.

Becker, P. J. \& Coppens, P. (1974a). Acta Cryst. A30, 148-153.

Becker, P. J. \& Coppens, P. (1975b). Acta Cryst. A31, 417-425.

Blessing, R. H. (1987). Crystallogr. Rev. 1, 3-58.

Blessing, R. H. (1995). Acta Cryst. A51, 33-38.

Blessing, R. H. (1997). J. Appl. Cryst. 30, 421-426.

Bojarowski, S. A., Kumar, P. \& Dominiak, P. M. (2017). Acta Cryst. B73, 598-609.

Bojarowski, S. A., Kumar, P. \& Dominiak, P. M. (2016). ChemPhysChem, 17, 2455-2460.

Bojarowski, S. A., Kumar, P., Wandtke, C. M., Dittrich, B. \& Dominiak, P. M. (2018). J. Chem. Theory Comput. 14, 6336-6345.

Brock, C. P., Dunitz, J. D. \& Hirshfeld, F. L. (1991). Acta Cryst. B47, 789-797.

Dadda, N., Nassour, A., Guillot, B., Benali-Cherif, N. \& Jelsch, C. (2012). Acta Cryst. A68, 452-463.

De Bruyne, B. \& Gillet, J.-M. (2020). Acta Cryst. A76, 1-6.

Dittrich, B., Hübschle, C. B., Luger, P. \& Spackman, M. A. (2006). Acta Cryst. D62, 1325-1335.

Dittrich, B., Hübschle, C. B., Messerschmidt, M., Kalinowski, R., Girnt, D. \& Luger, P. (2005). Acta Cryst. A61, 314-320.

Dittrich, B., Hübschle, C. B., Pröpper, K., Dietrich, F., Stolper, T. \& Holstein, J. (2013). Acta Cryst. B69, 91-104.

Dittrich, B., Koritsánszky, T. \& Luger, P. (2004). Angew. Chem. Int. Ed. 43, 2718-2721.

Dolomanov, O. V., Bourhis, L. J., Gildea, R. J., Howard, J. A. K. \& Puschmann, H. (2009). J. Appl. Cryst. 42, 339-341.

Domagała, S., Fournier, B., Liebschner, D., Guillot, B. \& Jelsch, C. (2012). Acta Cryst. A68, 337-351.

Dominiak, P. M., Volkov, A., Li, X., Messerschmidt, M. \& Coppens, P. (2007). J. Chem. Theory Comput. 3, 232-247.

Frisch, M. J., et al. (2009). GAUSSIAN09. Gaussian Inc., Wallingford, CT, USA.

Groom, C. R., Bruno, I. J., Lightfoot, M. P. \& Ward, S. C. (2016). Acta Cryst. B72, 171-179.

Gruza, B., Chodkiewicz, M. L., Krzeszczakowska, J. \& Dominiak, P. M. (2020). Acta Cryst. A76, 92-109.

Guillot, B., Viry, L., Guillot, R., Lecomte, C. \& Jelsch, C. (2001). J. Appl. Cryst. 34, 214-223.

Hansen, N. K. \& Coppens, P. (1978). Acta Cryst. A34, 909-921.

Holstein, J. J., Hubschle, C. B. \& Dittrich, B. (2012) CrystEngComm 14, 2520-2531. 
Jarzembska, K. N. \& Dominiak, P. M. (2012). Acta Cryst. A68, 139-147.

Jayatilaka, D. \& Dittrich, B. (2008). Acta Cryst. A64, 383-393.

Jayatilaka, D. \& Grimwood, D. J. (2001). Acta Cryst. A57, 76-86.

Jelsch, C., Guillot, B., Lagoutte, A. \& Lecomte, C. (2005). J. Appl. Cryst. 38, 38-54.

Jha, K. K., Gruza, B., Kumar, P., Chodkiewicz, M. L. \& Dominiak, P. M. (2020). Acta Cryst. B76, 296306.

Johnson, C. K. (1969). Acta Cryst. A25, 187-194.

Keen, D. A., Gutmann, M. J. \& Wilson, C. C. (2006). J. Appl. Cryst. 39, 714-722.

Kendall, R. A., Dunning, J. T. H. \& Harrison, R. J. (1992). J. Chem. Phys. 96, 6796-6806.

Koritsanszky, T., Volkov, A. \& Chodkiewicz, M. (2012). Struct. Bond. 147, 1-25.

Kuhs, W. F. (1983). Acta Cryst. A39, 148-158.

Kumar, P., Bojarowski, S. A., Jarzembska, K. N., Domagała, S., Vanommeslaeghe, K. Jr, MacKerell, A.

D. Jr \& Dominiak, P. M. (2014). J. Chem. Theory Comput. 10, 1652-1664.

Kumar, P., Gruza, B., Bojarowski, S. A. \& Dominiak, P. M. (2019). Acta Cryst. A75, 398-408.

Lee, C., Yang, W. \& Parr, R. G. (1988). Phys. Rev. B, 37, 785-789.

Lübben, J., Wandtke, C. M., Hübschle, C. B., Ruf, M., Sheldrick, G. M. \& Dittrich, B. (2019). Acta Cryst. A75, 50-62.

Madsen, A. Ф. (2006). J. Appl. Cryst. 39, 757-758.

Malaspina, L. A., Wieduwilt, E. K., Bergmann, J., Kleemiss, F., Meyer, B., Ruiz-López, M. F., Pal, R., Hupf, E., Beckmann, J., Piltz, R. O., Edwards, A. J., Grabowsky, S. \& Genoni, A. (2019). J. Phys. Chem. Lett. 10, 6973-6982.

Malińska, M., Jarzembska, K. N., Goral, A. M., Kutner, A., Woźniak, K. \& Dominiak, P. M. (2014). Acta Cryst. D70, 1257-1270.

Mandal, S. K., Guillot, B. \& Munshi, P. (2020). CrystEngComm 22, 4363-4373.

Meindl, K. \& Henn, J. (2008). Acta Cryst. A64, 404-418.

Nassour, A., Domagala, S., Guillot, B., Leduc, T., Lecomte, C. \& Jelsch, C. (2017). Acta Cryst. B73, 610625.

Perdew, J. P. (1986). Phys. Rev. B, 33, 8822-8824.

Petříček, V., Dušek, M. \& Palatinus, L. (2014). Z. Kristallogr. Cryst. Mater. 229, 345-352.

Řezáč, J., Riley, K. E. \& Hobza, P. (2011a). J. Chem. Theory Comput. 7, 3466-3470.

Řezáč, J., Riley, K. E. \& Hobza, P. (2011b). J. Chem. Theory Comput. 7, 2427-2438.

Sanjuan-Szklarz, W. F., Woińska, M., Domagała, S., Dominiak, P. M., Grabowsky, S., Jayatilaka, D., Gutmann, M. \& Woźniak, K. (2020). IUCrJ, 7, 920-933.

Sanjuan-Szklarz, W. F., Hoser, A. A., Gutmann, M., Madsen, A. Ø. \& Woźniak, K. (2016). IUCrJ, 3, 6170.

Scheringer, C. (1985). Acta Cryst. A41, 79-81.

Sheldrick, G. M. (2008). Acta Cryst. A64, 112-122.

Sheldrick, G. M. (2015a). Acta Cryst. A71, 3-8.

Sheldrick, G. M. (2015b). Acta Cryst. C71, 3-8.

Stewart, R. F. (1976). Acta Cryst. A32, 565-574.

Su, Z. \& Coppens, P. (1998). Acta Cryst. A54, 646-652

Tanaka, K. (2018). Acta Cryst. A74, 345-356.

Volkov, A., Koritsańszky, T. \& Coppens, P. (2004a). Chem. Phys. Lett. 391, 170-175.

Volkov, A., Li, X., Koritsanszky, T. \& Coppens, P. (2004b). J. Phys. Chem. A, 108, 4283-4300.

Volkov, A., Macchi, P., Farrugia, L. J., Gatti, C., Mallinson, P., Richter, T. \& Koritsanszky, T. (2006b). XD2006. Middle Tennessee State University, USA, Università di Milano and CNR-ISTM Milano, Italy, University of Glasgow, Scotland, State University of New York at Buffalo, USA, and Freie Universität Berlin, Germany. 
Volkov, A., Macchi, P., Farrugia, L., Gatti, C., Mallinson, P., Richter, T. \& Koritsanszky, T. (2016).

XD2016 - A Computer Program Package for Multipole Refinement, Topological Analysis of

Charge Densities and Evaluation of Intermolecular Energies from Experimental and Theoretical Structure Factors.

Volkov, A., Messerschmidt, M. \& Coppens, P. (2007). Acta Cryst. D63, 160-170.

Zarychta, B., Lyubimov, A., Ahmed, M., Munshi, P., Guillot, B., Vrielink, A. \& Jelsch, C. (2015). Acta Cryst. D71, 954-968.

Zarychta, B., Pichon-Pesme, V., Guillot, B., Lecomte, C. \& Jelsch, C. (2007). Acta Cryst. A63, 108-125. 

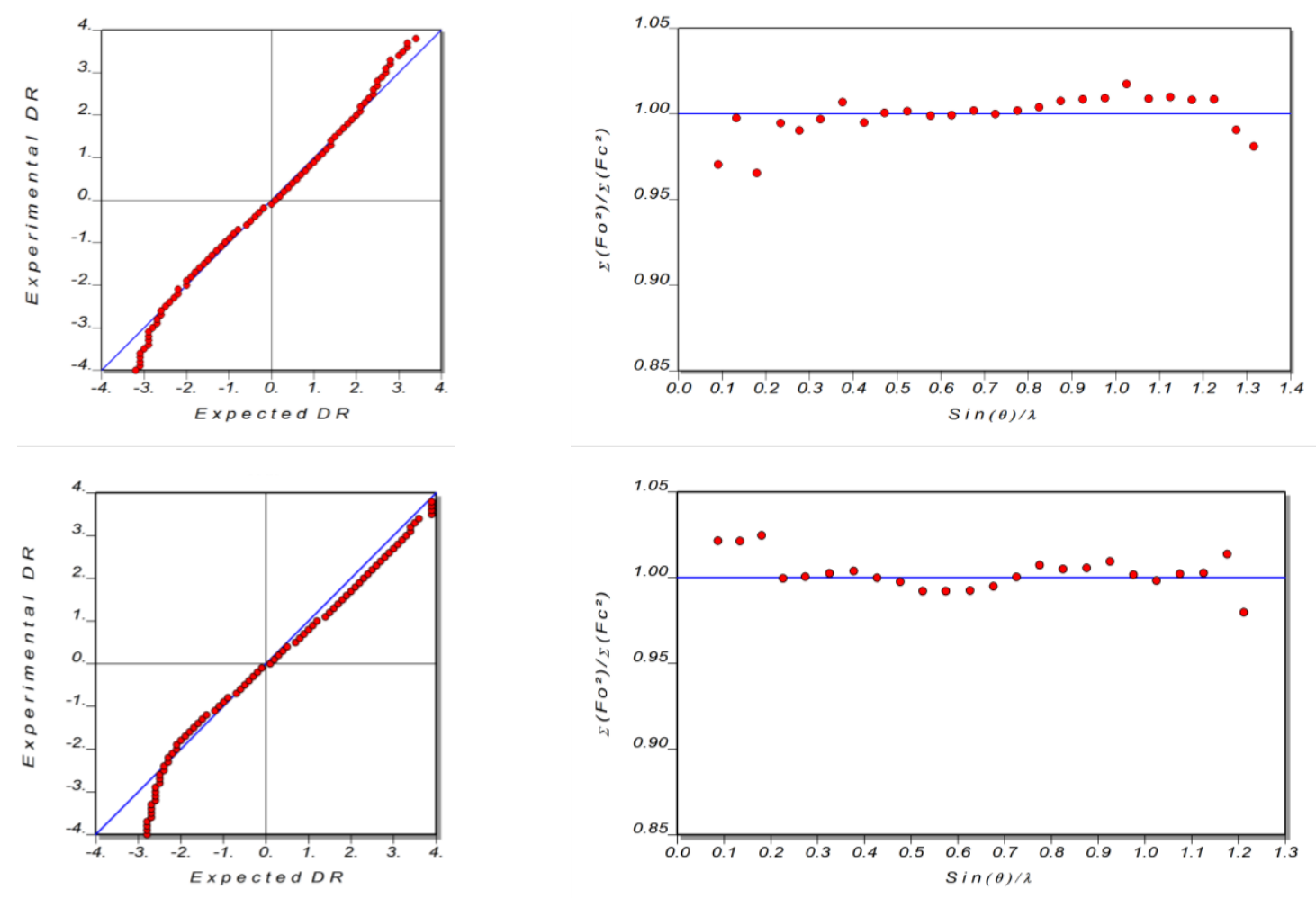

Figure 1S. Normal probability plots of $F^{2}$ residuals (left) and scale plots (right) from multipole model refinement for cyanuric acid dihydrate (top) and triptycene (bottom). 

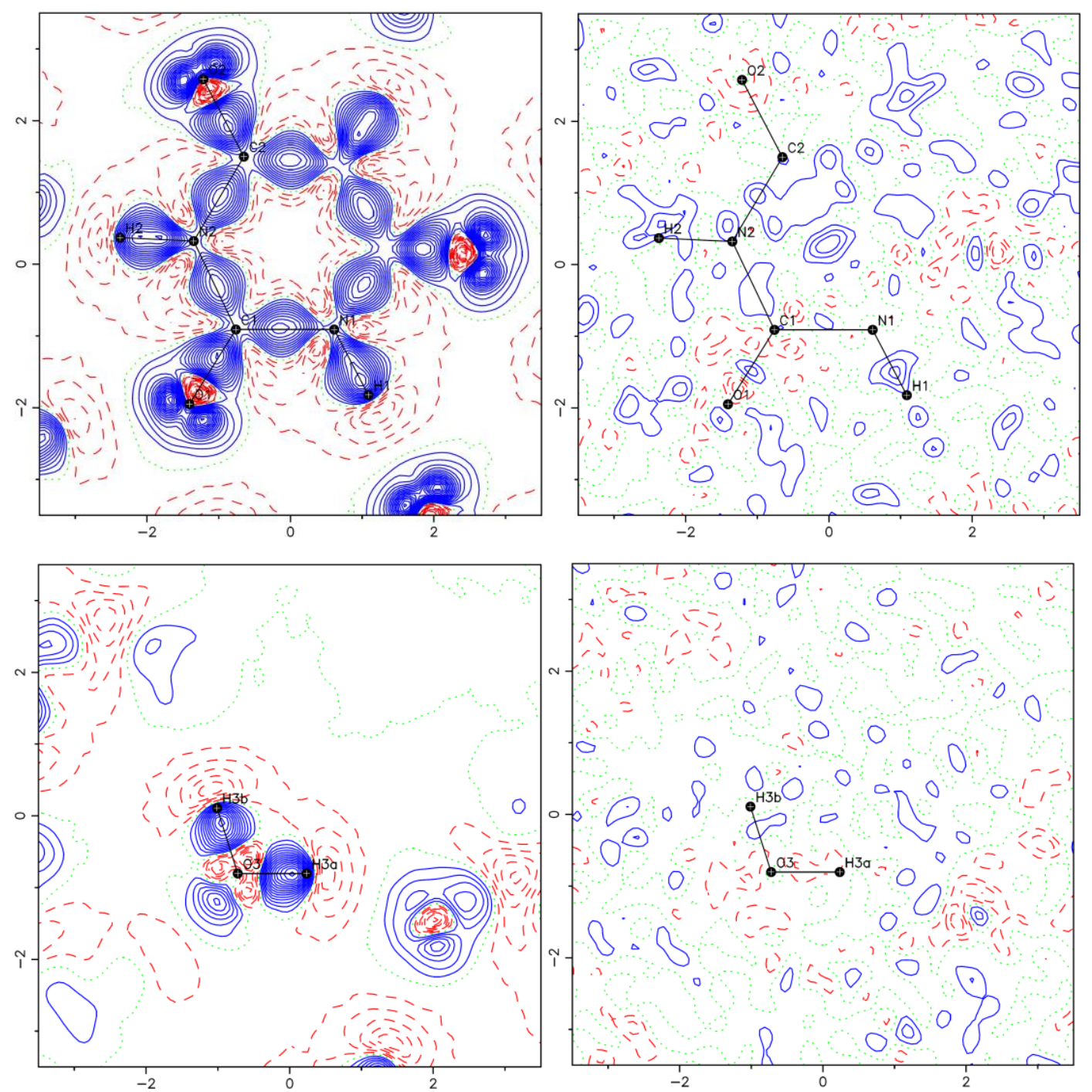

Figure 2S. Static deformation electron density (left) and residual electron density (right) Fourier maps computed for all data from multipole model refinement for cyanuric acid dihydrate. Contouring at 0.05 e. $\AA^{-3}$; blue solid lines - positive values, red dashed lines - negative values.
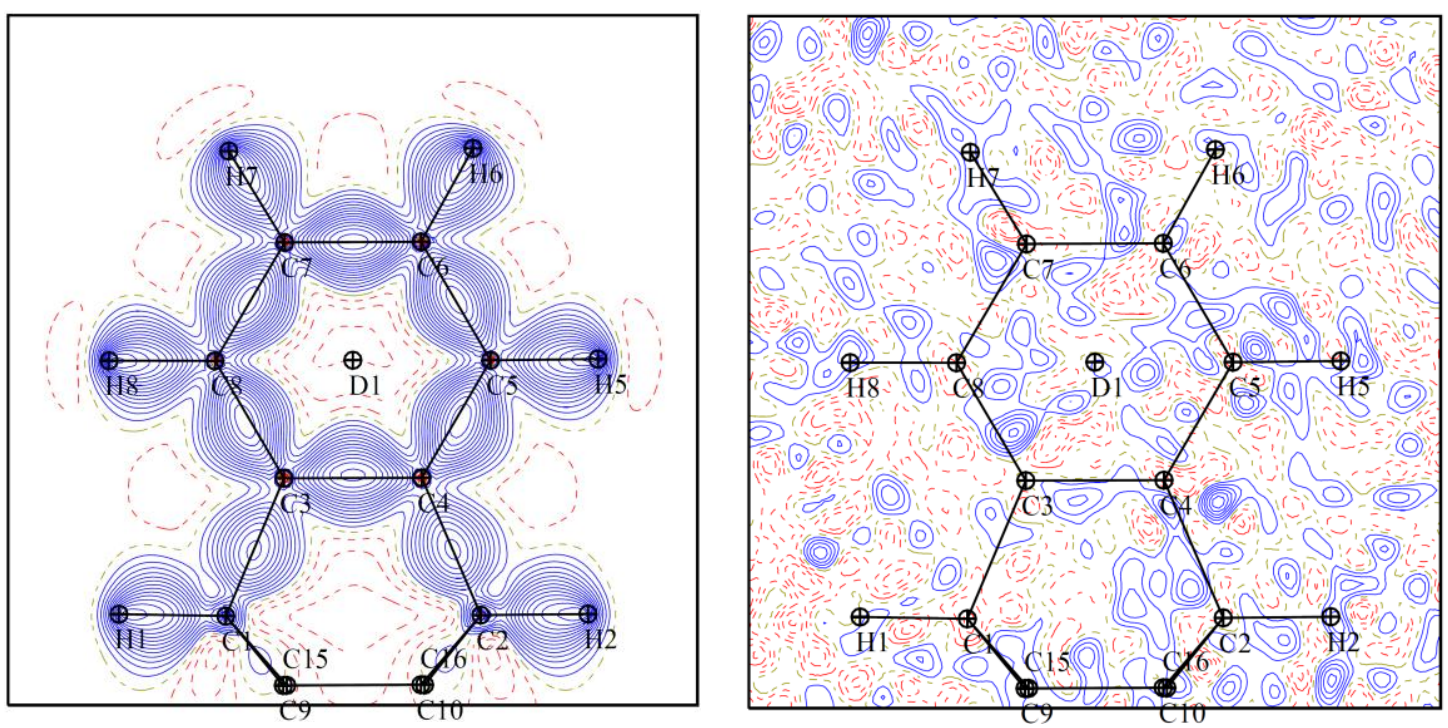

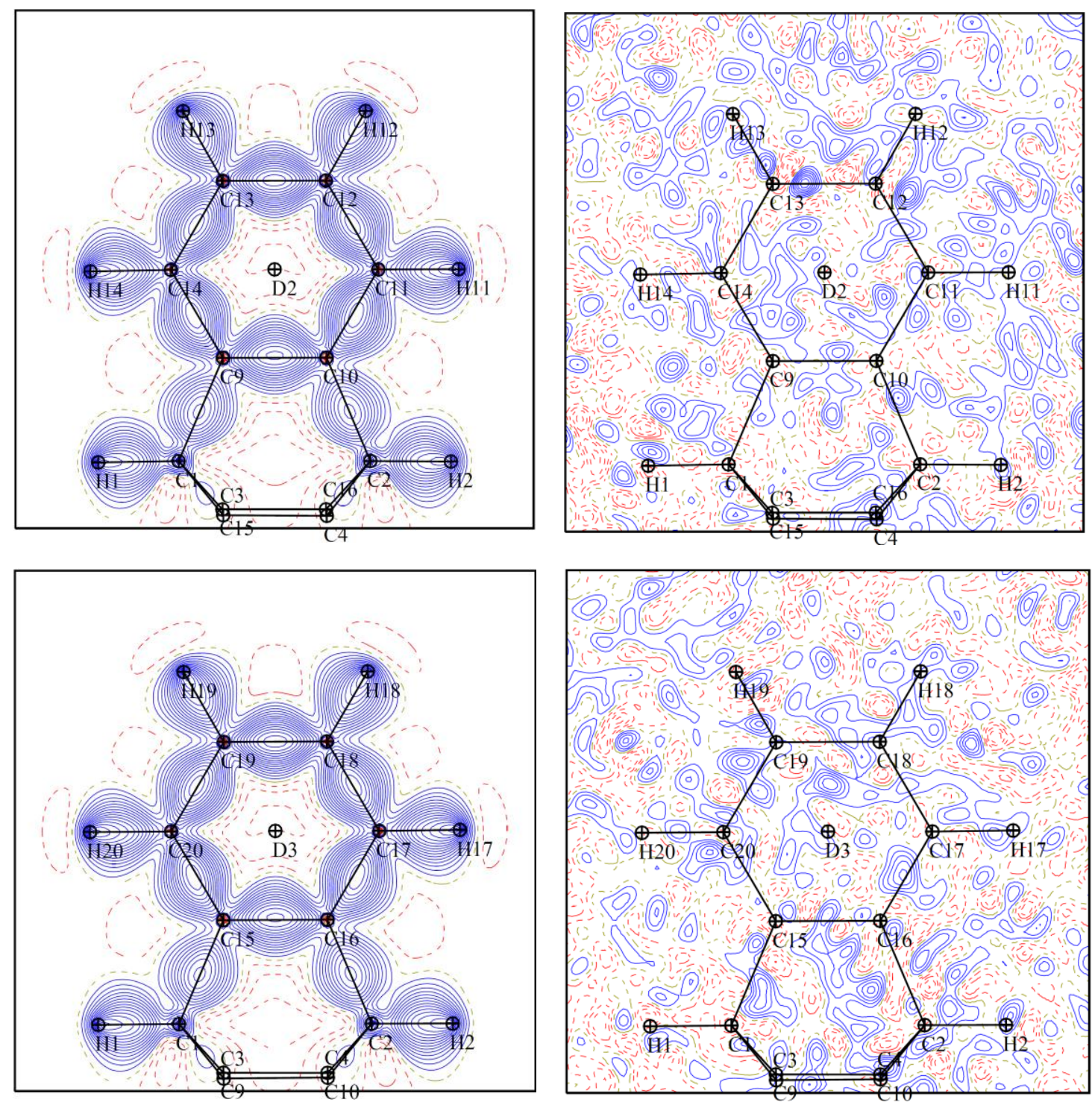

Figure 3S. Static deformation electron density (left) and residual electron density (right) Fourier maps computed for all data from multipole model refinement for triptycene. Contouring at $0.05 \mathrm{e} \cdot \AA^{-3}$; blue solid lines - positive values, red dashed lines - negative values.
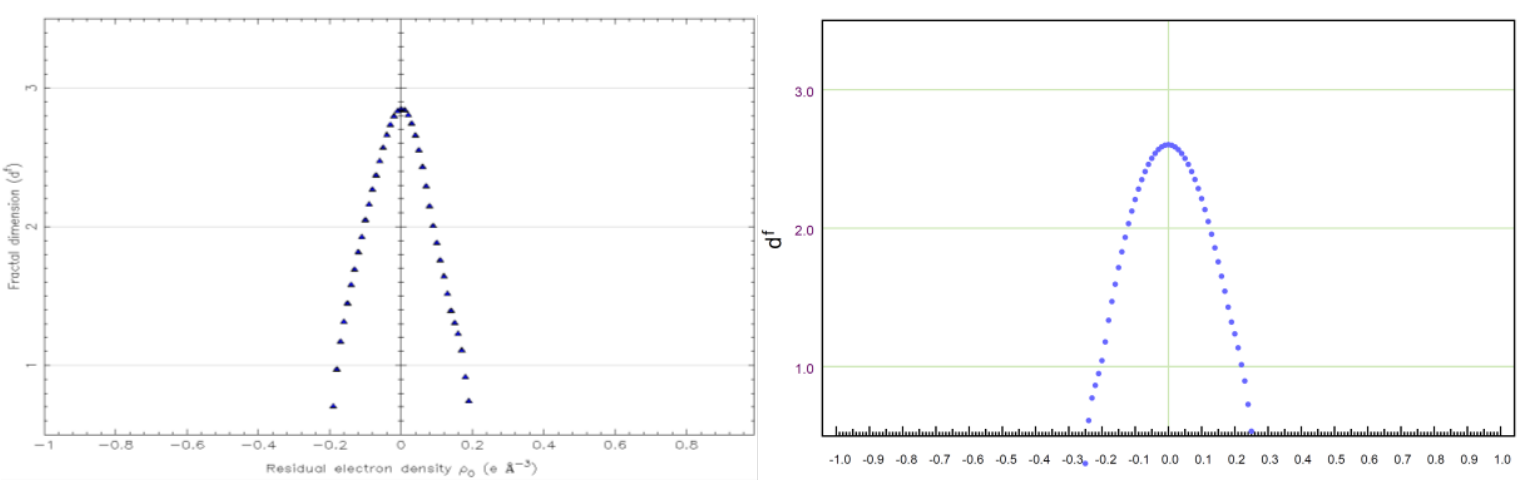

Figure 4S. Fractal dimension plots for residual electron density from multipole model refinement for cyanuric acid dihydrate (left) and triptycene (right). 